\title{
Biointerfaces
}

\section{Cell Surface Engineering to Control Cellular Interactions}

\author{
Catarina A. Custódio ${ }^{[a, b]}$ and João F. Mano*[a,b]
}

\begin{abstract}
Cell surface composition determines all interactions of the cell with its environment, thus cell functions such as adhesion, migration and cell-cell interactions can potentially be controlled by engineering and manipulating the cell membrane. Cell membranes present a rich repertoire of molecules, therefore a versatile ground for modification. However the complex and dynamic nature of the cell surface is also a major challenge for cell surface engineering that should also involve strategies compatible with cell viability. Cell surface engineering by selective chemical reactions or by the introduction of exogenous targeting ligands can be
\end{abstract}

a powerful tool for engineering novel interactions and controlling cell function. In addition to chemical conjugation and modification of functional groups, ligands of interest to modify the surface of cells include recombinant proteins, liposomes or nanoparticles. Here, we review recent efforts to perform changes to cell surface composition. We focus on the engineering of the cell surface with biological, chemical or physical methods to modulate cell functions and control cell-cell and cell-microenvironment interactions. Potential applications of cell surface engineering are also discussed.

\section{Introduction}

Living cells are sensitive to their environment. This means that they detect and respond to events in their surrounding environment. $^{[1]}$

The surface of cells contains a diversity of receptors that serve as the primary conduits for transmission of environmental information into the cell's signaling network. (Figure 1). Also, many of these surface ligands may be associated with other cells or with extracellular materials, regulating extracellular communication. Thus, different strategies or chemical reaction methodologies can be used to functionalize cell membranes.

Natural extracellular matrix (ECM) serves as both a structura scaffold and a substrate for the display of signaling ligands. ${ }^{[2,3]}$ During the past decades several works have been focused on developing synthetic materials to control cell behavior, mimicking or reconstituting the ECM in various ways. ${ }^{[4]}$ In a similar approach, recently Salmeron-Sanchez and co-workers proposed the concept of living biointerfaces to control cell fate. ${ }^{[5]}$ They

\section{[a] C. A. Custódio, J. F. Mano}

3B's Research Group - Biomaterials, Biodegradables and Biomimetics University of Minho

Headquarters of the European Institute of Excellence of Tissue Engineering and Regenerative Medicine

Avepark - Parque de Ciência e Tecnologia, Zona Industrial da Gandra 4805-017 Barco GMR (Portugal)

[b] C. A. Custódio, J. F. Mano

ICVS/3B's PT Government Associated Laboratory Braga/Guimarães (Portugal)

(iD The ORCID identification number(s) for the author(s) of this article can be found under http://dx.doi.org/10.1002/cnma.201600047.

This manuscript is part of a Special Issue on Nanobiointerfaces. A link to the Table of Contents will appear here once the Special Issue is assembled. investigated the potential of a living interface based on L. lactis expressing a fibronectin fragment as a membrane protein to enhance cell adhesion and direct cell differentiation. Conversely, strategies to engineer and manipulate cell-surface interactions involving chemical modification of the cell membrane, or using the cellular adhesion machinery itself through genetic engineering only recently started to be explored.
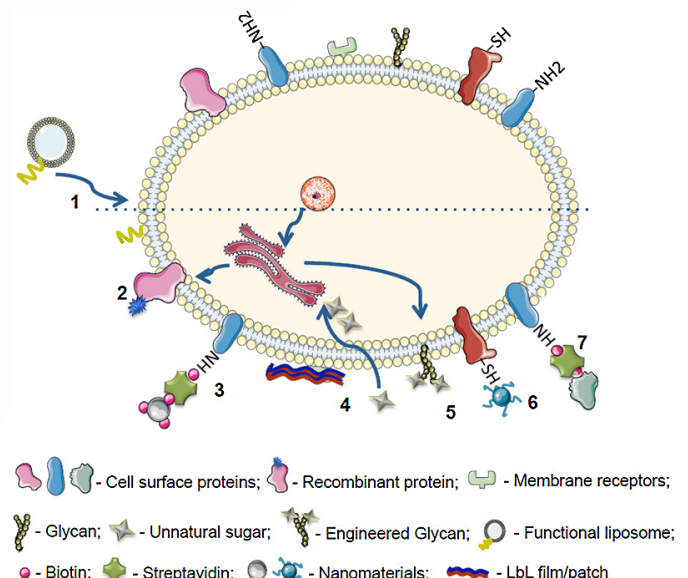

Figure 1. Representation of the cell surface structures and the principal methodologies used for cell engineering: 1) Chemically modified liposomes fuse with the cell membrane, incorporating functional groups. 2) Site-specific protein modification via genetic engineering. 3) Covalent immobilization of biotin to membrane proteins via amine group followed by streptavidinbiotin binding of nanomaterials. 4) LbL technique to deposit functional, heterostructured polymer films at the cell's surface. 5) Metabolic labeling of glycans with synthetic sugars. 6) Covalent conjugation of target molecules and/ or nanomaterials via thiol groups. 7) Covalent immobilization of biotin to membrane proteins via amine group followed by streptavidin-biotin binding of target molecules. 
In this review, we will focus on the advanced techniques to engineer cell surfaces, describe their potential and challenges, highlighting the strategies that have been explored to regulate cell-cell and cell-extracellular matrix interactions.

The cell membrane is a highly complex and dynamic environment comprising lipids, proteins and carbohydrates, which mediate extracellular communication. ${ }^{[6]}$ This rich repertoire of molecules presents an excellent opportunity to engineer the cell membrane and a powerful tool to manipulate interactions between cells and the surrounding environment. However, cell surface engineering is particularly challenging due to the fact that the cell membrane in not a static structure. ${ }^{[7-9]}$ It should also be noted that any process for cell surface engineering must be performed using minimal alterations to the biological environment of living cells, as slight alterations of $\mathrm{pH}$, temperature, ionic strength and osmolality $\square$ can have a significant influence $\mathbf{\square}$.

Cells can be engineered by chemical modifications in the cell membrane through chemical conjugation or non-covalent interactions. Furthermore, cells can be tailored with nanomaterials or coated using layer-by-layer (LbL) strategies for engineering novel interactions and controlling cell function. Some of these engineering techniques still require optimization to improve the efficacy and targeting effectiveness while minimizing any loss of cell function. In this review we will first describe which molecules of interest comprise the membrane and how they are arranged, then we summarize key methodologies used to manipulate the surface of living cells. We will then discuss how these cell modifications can be applied to control cell function or enhance the therapeutic potential of cellular products. Finally, we will outline future trends and perspectives of this breakthrough field.

\section{The cell surface}

The interactions of cells with the surrounding environment are mediated by the cell membrane, thus it is worth considering the biomolecular composition of the membrane and how these molecules are arranged. Cell membranes are composed of a lipid bilayer, containing proteins that span the bilayer on either side of the two leaflets designed to perform the functions cell require. ${ }^{[6]}$ Proteins are central molecules in cell-ECM interactions, typically through the creation of attachment points linking the cytoskeleton to extracellular binding sites. The capacity to manipulate cells interactions with the surrounding environment will certainly be dependent on our ability to control the function of these proteins. Integrins are a superfamily of transmembrane cell adhesion proteins that bind to ECM ligands, cell-surface ligands, and soluble ligands. Syndecans and lectins are a family of transmembrane core proteins that act synergistically with integrins as co-receptors for ECM proteins that bind specific carbohydrates. Many membrane proteins and lipids are conjugated to polysaccharides, which comprise the glycocalyx, or cell coat, of all cells. ${ }^{[9]}$ Cell surface glycans also play crucial roles in various physiological events involving cell surface recognition. ${ }^{[10,11]}$ Unfortunately, the heterogeneous nature of cell surfaces, particularly with re- spect to glycoconjugate structures, has frustrated molecularlevel studies of glycan function.

Cell surface engineering is challenging due to the fact that the plasma membrane is a dynamic structure: both lipid and protein components of the membrane are continuously internalized, displaced, degraded, and replaced by de novo synthesis. ${ }^{[12]}$ Chemical conjugation and/or insertion of target molecules, nanomaterials or patches within the plasma membrane, are some particularly important trends in cell engineering that will be discussed below. These strategies to decorate cell's surface would enable a close control in cell behavior, from cell adhesion to cell migration, proliferation or differentiation.

\section{Strategies for Cell Surface Bioengineering}

\section{Genetic engineering}

Genetic engineering is well-established as a robust and highly versatile methodology employing the cell's biosynthetic machinery to modify the genetic programming of cells. ${ }^{[13]}$ The modulation of cell surface receptor expression through genetic modification was recently exploited to alter cell surfaces, remodeling extracellular communication. The base of genetic engineering is the inclusion of exogenous genetic material into the cell to express or regress specific cell surface molecules to achieve the preferred outcome. ${ }^{[8,14]}$ Some recent studies have focused on the expression of key cell surface receptors involved in stem cell recruitment and migration. ${ }^{[15]}$ Genetic manipulation of integrin expression in cells could significantly improve cell engraftment, increasing the efficiency of cell therapy.

João F. Mano is a Professor at the Chemistry Department of University of Aveiro and principal investigator at CICECO. His current research interests include the use of biomaterials and cells towards the development of transdisciplinary concepts for biomedical applications, especially aimed at being used in regenerative/personalised medicine. In particular, he and his group have been developing materials, mainly derived from biodegradable polymers, with stimuli-responsive or bio-instructive behaviour, or biomimetic and nano/ micro-technology approaches applied to biomaterials and surfaces.

Catarina A. Custódio is graduated in Applied Chemistry by the Faculty of Sciences and Technology of the New University of Lisbon and obtained her PhD on Tissue Engineering Regenerative Medicine and Stem Cells in the University of Minho and in cooperation with the Max Planck Institute for Polymer Research in Mainz. She is currently working as a postdoctoral research rat University of Aveiro. Her research interests lie at the development of novel bioactive hydrogels and new approaches to control cell behavior through the nanoscale (bio)engineering of materials surfaces.
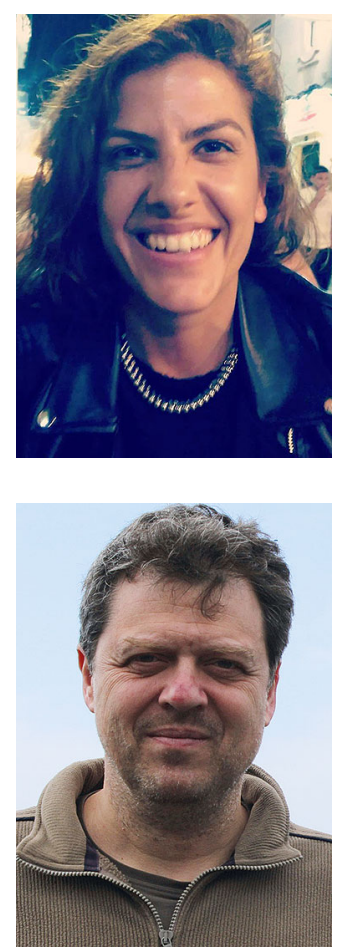
Mrksich and co-workers have used cell engineering and synthetic surface chemistry as complementary strategies to promote unique specific ligand-receptor interactions. ${ }^{[16]}$ They constructed a chimeric receptor that contains the intracellular and transmembrane domains of $\beta 1$ integrin combined with other specific domains providing a new specificity for the binding of the receptor to ECM. Cells expressing the modified receptor adhered and spread selectively on the target substrates. The same group described an approach for integrating cellular activities and electrical processes in an underlying substrate by cell surface engineering. ${ }^{[17]}$ Using genetic manipulation they introduced enzymes at the cell surface that will modify electroactive monolayers, enabling electronic transduction of biological activity. Genetic manipulation was also used to introduce bio-orthogonal reactive groups into cell surface proteins, creating sites for selective modification of cells. For example, Ting and co-workers developed a robust methodology to label site specifically cell surface proteins with biotin groups. ${ }^{[18]}$ They genetically attach a specific peptide to either terminus of the protein of interest and add recombinant enzyme biotin ligase that site-specifically biotinylates a lysine side chain within the peptide to the cell medium. These biotin groups can then be targeted with streptavidin conjugates. The same enzyme catalyzes ligations that also permit the derivatization of membrane proteins with ketone groups, which further extends the spectrum of possible conjugates. ${ }^{[19]}$ Membrane associated proteins can also be site-specifically modified by using a genetically encoded aldehyde tag. ${ }^{[20]}$ Proteins bearing this aldehyde tag are then chemically modified by selective reaction with hydrazideor aminooxy-functionalized reagents. The precise chemical control offered by the aldehyde tag method should enable the development of new protein products for research and therapeutic purposes. Despite the great achievements and promising results, genetic manipulation of cells is technically challenging dealing with regulatory and safety issues. To overcome these issues there is growing interest in devising novel bioconjugation, protein engineering, chemistry, and material science approaches for cell surface engineering. ${ }^{[21]}$

\section{Chemical modification}

In contrast to genetic engineering that is mostly used to manipulate proteins at the cell's surface, chemical modification may be used to manipulate lipids, proteins or glycans. Chemical functional groups naturally present on this biomolecules at cell surface are appealing sites for functionalization using covalent conjugation. The most commonly used chemical groups include, amines, sulfhydryl, carboxyl and carbonyl groups present in proteins and other cell surface molecules. It is worth to note that chemical reactions on the cell surface should be selective without any nonspecific modifications and performed under mild conditions.

Amine groups are widely used for chemical modification of cell membranes due to well-established protocols, easily available conjugation linkers and mild reaction conditions. The most common reaction to modify primary amines involves reaction with $\mathrm{n}$-hydroxysuccinimide (NHS) ester. This strategy was used for the direct coupling of succinimidyl ester-functionalized polyethylene glycol (PEG) to cell surface amines of pancreatic islets. ${ }^{[2]}$ Such modification has been studied toward preventing immune responses of host for successful islet transplantation

Using a similar chemistry, biotin has been covalently conjugated on the cell membrane that can be subsequently functionalized through strept(avidin). Such functionalization allows for the immobilization of different functional molecules. Salem and co-workers reported a quick cell surface functionalization with biotin for the preparation of synthetic biodegradable microparticle-biological-transfected-cell hybrids. ${ }^{[23]}$ Microparticles displaying a biotin-enriched surface can bound to the biotinylated cell surface with avidin as a bridging protein (Figure 2). The microparticles can easily be loaded with proteins, immunostimulatory molecules, or growth factors. This system has therefore significant potential for multifunctional drug delivery applications. This approach has been also used to functionalize mesenchymal stem cells (MSCs) with Sialyl Lewis X (SLeX) or platelet-derived growth factor (PDGF) sensing aptamer. ${ }^{[24,25]}$

Thiols, present in the cysteine residues of proteins, are another important chemical compound used for cell surface functionalization. A major advantage of thiol-based functionalization strategies is the wide range of available reagents for labeling this functional group. Cell surface thiols are present either in oxidized disulfide bridges or in reduced thiol groups. The most widely used covalent reactions involve maleimide-activated molecules that specifically react with thiol groups. For example, maleimide-functionalized PEG was used for chemical modification of red blood cells (RBCs) to camouflage the blood group antigens from their antibodies. ${ }^{[26]}$ The protocol involves the modification of a set of surface amino groups of protein as
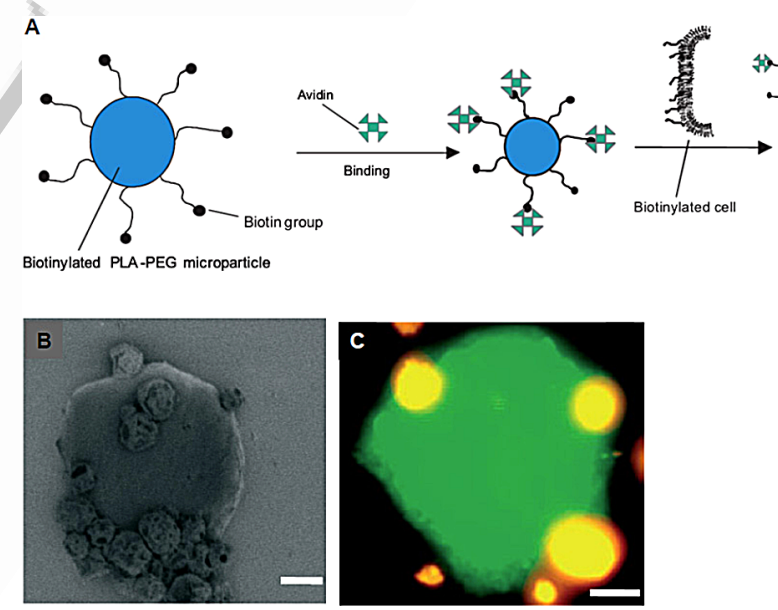

Figure 2. A) Schematic depiction of the self-assembly of microparticle-cell hybrids B) SEM image of self-assembled microparticle-cell hybrids (scale bar $=1.5 \mu \mathrm{m}$ ), C) fluorescence microscopy overlay image ofHEK293 cells transfected with green fluorescent protein and assembled with PLA-PEG-biotin microparticles loaded with rhodamine 123 (555/ $580 \mathrm{~nm}, 494 / 518 \mathrm{~nm}$, scale bar $=1.5 \mu \mathrm{m}$ ). (Reprinted with permission from [23]. Copyright $\odot 2008$ Wiley-VCH Verlag GmbH \& Co. KGaA.) 
maleimide-reactive thiols (thiolation) followed by the conjugation of maleimide PEG to these sites. In another study Stephan and co-workers have conjugated nanoparticles functionalized with thiol-reactive maleimide head groups on cell membranes via maleimide-based chemistry for actively targeted drug delivery. ${ }^{[27]}$

A few strategies for engineering cell-surface glycans have been reported for applications such as studying cell-surface phenomena, including receptor clustering. ${ }^{[28]}$ Chen and coworkers proposed the cell-specific metabolic glycan labeling using ligand-targeted liposomes to deliver unnatural sugars to target cells in a cell-surface receptor dependent manner. ${ }^{[29]}$ The delivered sugars are metabolically incorporated into cell-surface glycans. Using a similar approach, Hsieh-Wilson and coworkers recently developed a method to display specific sulfated glycosaminoglycans (GAGs) structures on cell surfaces using a liposomal fusion strategy (Figure 3). ${ }^{[30]}$ To promote membrane fusion and surface presentation of the glycans they used cationic 1,2-dioleoyl-3-trimethylammonium-propane chloride (DOTAP) and neutral 1,2-dioleoyl-sn-glycero-3-phosphatidylethanolamine (DOPE) as primary lipids to fabricate the liposomes. It was shown that tailoring neuron cells with chondroitin sulfate GAGs activates growth factor-mediated signaling pathways and enables the fine-tuned modulation of neuronal growth.

The cell membrane is complex and rich in chemical groups but some functionalities are not available for direct covalent reaction under feasible conditions. ${ }^{[31]}$ To enhance the capability of covalent modification on cell surfaces, specific functionalities may be introduced on cell membrane. Biomimetic reactions (e.g., biotin-(strept)avidin and antibody-antigen) are appealing for cell modification, as cells may recognize those reactions to be part of natural processes. A pioneering study from Bertozzi and co-workers reported the use of cell metabolism to introduce a reactive functional group into the cell surface-associated sialic acid residues. ${ }^{[32]}$ Remodeling of cell surface was performed by the condensation of ketones with aminooxy or hydrazide reagents, resulting in the introduction of ketone

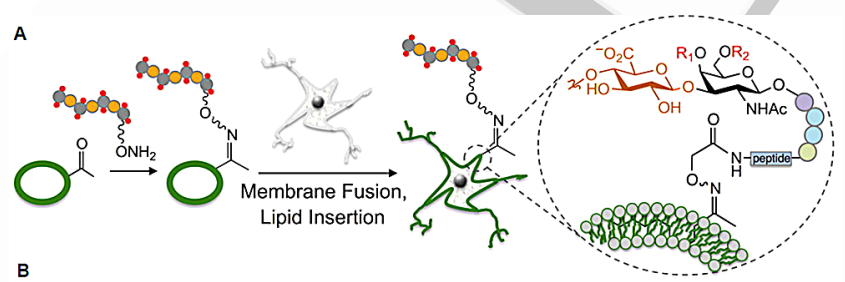

B
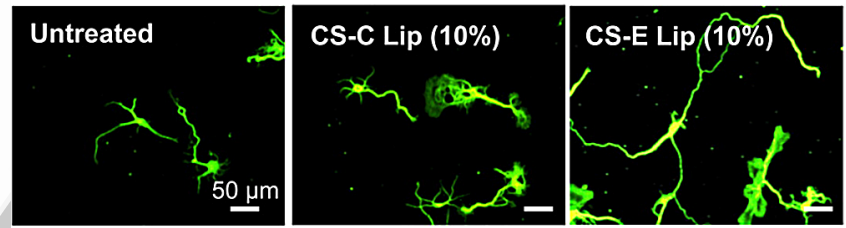

Figure 3. A) Strategy to remodel cell surfaces with CS GAGs and control signaling pathways. B) Representative images of neurite outgrowth for neurons treated with unmodified liposomes or liposomes displaying CS-A-, CS-C-, or CS-E-enriched polysaccharides. (Reprinted with permission from [30]. Copyright $\odot 2014$ American Chemical Society.) groups. Because the display of ketone groups is achieved through the action of the biosynthetic machinery it may be used to engineer cell surface in vivo.

Francis and co-workers have developed a synthetic method based on the introduction of specific chemical handles onto cell surfaces by using metabolic oligosaccharide engineering. ${ }^{[33]}$ Azides were incorporated into cell surface glycans by the introduction and further metabolism of the unnatural azido sugars. These unnatural sugars are metabolized to the corresponding azide within membrane associated glycans. This functional group was then used to include an oligonucleotide sequence at the cell's surface. Modified single strand (ssDNA) was prepared with a phosphine group through the reaction of a 5 amine-modified ssDNA with a phosphine pentafluorophenyl ester. The phosphine-ssDNA conjugate undergoes the Staudinger ligation with the azide to form the expected amidelinked product. ${ }^{[34]}$

\section{Electrostatic interactions}

Layer-by-layer (LbL) self-assembly is a thin film fabrication technique with physiological versatility that works by depositing alternating layers of materials usually exhibiting opposite charge. ${ }^{[35]}$ After the interaction between the negatively charged cell membrane and a positively charged polyelectrolyte, the cell surface may be turned positive. After a certain number of cycles a multilayered structure with tunable robustness may be generated, creating a defined microenvironment for cell differentiation and proliferation, as well as a model for studies in cell biology. This method has been used for a variety of cell types or living tissues such as pancreatic islets and using diverse natural polymers. ${ }^{[36,37]}$

LbL of alginate and chitosan-graft-phosphorylcholine was used to coat RBCs to form a barrier that prevents the recognition of antigenic sites by antibodies. ${ }^{[38]}$ In addition to the immune protection the LbL self-assembly of polyelectrolytes on RBCs preserved their viability and functionality. Mouse MSCs have been individually encapsulated by polyelectrolyte layers of poly (L-lysine) and hyaluronic acid using LbL resulting in a capsule consisting of nanolayers of thickness around 6$9 \mathrm{~nm}$, cell viability of encapsulated cells was demonstrated for up to one week. ${ }^{[39]}$ In a more recent study LbL using gelatin and alginate as polycation and polyanion was used to encapsulate single neural stem cells (NSCs). ${ }^{[40]}$ The enhancement of proliferation was found in NSCs encapsulated with IGF1 loaded materials, demonstrating the successful application of the encapsulation model in improving the properties of NSCs (Figure 4). In addition, the survival rate of NSCs was maintained for at least 10 days. The external coating of the multilayers can be in principle designed to increase the functionality of the cells, including targeting capability, adhesion to surfaces and other cells. Moreover, it could provide assembling capacity to generate higher-scale organized structures using the coated cells as building blocks.

The LbL technique shows great potential for cell modification, as is easy to perform, may be performed in mild conditions and does not require covalent conjugation. 


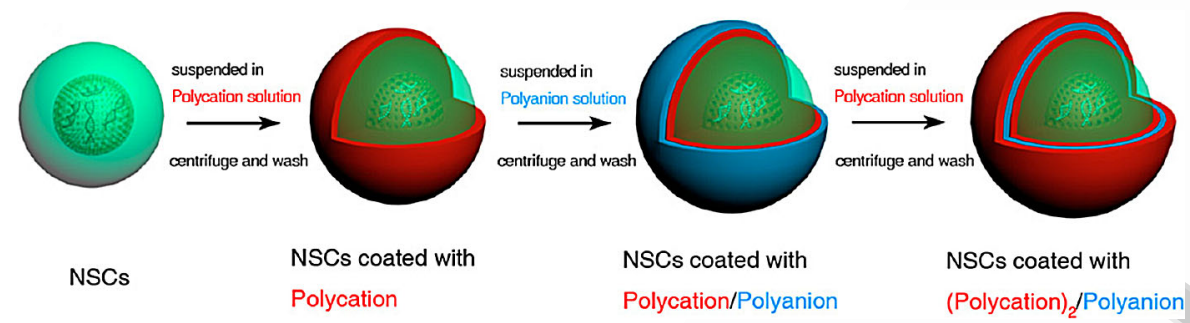

Figure 4. Illustration of the major steps involved in the LbL encapsulation: NSCs were first suspended in the polycation solution and then centrifuged and washed. The polycation layer was supposed to be on the cell surface. Next, the polycation-coated NSCs were put in the polyanion solution to add a second layer. The LbL encapsulation would be completed after several repetitions of this process. (Reprinted with permission from [40]. Copyright @ 2014 American Chemical Society.)

\section{Nanopatches and Nanoparticles}

Synthetic nanomaterials have a significant role to play in cell surface engineering, due to their unique properties and ability to provide functionality beyond that achievable by single molecules. Alternatively to the total and uniform cell surface modification achieved using LbL technique, the modification of a portion of the cell surface using nanoparticles or nanopatches offers other possibilities of tailoring the cell surface. However, nanoscale objects are easily internalized by cells limiting the tailoring of the surface of the cells. The reduction of internalization is therefore the key to construct effective modification of a portion of the cell membrane. For example Rubner and co-workers studied the influence of cell membrane-attached multilayered patches containing superparamagnetic nanoparticles onto lymphocytes surfaces on cell viability and migration (Figure 5A). ${ }^{[41]}$ The functional multilayered patches were successfully attached to a fraction of the surface area of living individual lymphocytes. Surface-modified cells remain viable at least $48 \mathrm{~h}$ following attachment of the functional patch. Moreover patches carrying magnetic nanoparticles allow the cells to be spatially manipulated using a magnetic field, extending their use in regenerative medicine. ${ }^{[42]}$ Using the biomolecular recognition through the avidin-biotin interaction, Anderson and co-workers developed nanoparticulate cellular patches that were anchored on MSCs possessing biotinylated plasma membrane. These nanoparticulate patches remain on the membrane of cells for days and provide a new system for cellmediated tumoritropic drug delivery. ${ }^{[43]} \mathrm{A}$ different method was recently proposed by Guan and co-workers, based on the use of microcontact printing ( $\mu \mathrm{CP})$ of polymeric biomaterials for functionalizing and assembling live cells (Figure $5 \mathrm{~B}$ ). ${ }^{[4]]}$ The method started by spin-coating a thin layer of a temperaturesensitive sacrificial layer on a glass slide. A stamp coated with the material for functionalization is then placed in contact with the sacrificial layer. Cells are seeded on the slide at $37^{\circ} \mathrm{C}$ to allow immobilization of the cells to the printed biomaterials; subsequently a decrease to room temperature allows the release of cell-biomaterial complexes. This strategy was then to generate cell-biomaterial complexes consisting of microcontact-printed structures of various sizes, shapes and materials. We envisage that such methodologies could be combined with other surface modification of cells, as upon adhesion the dorsal portion of the cells is susceptible to be modified.
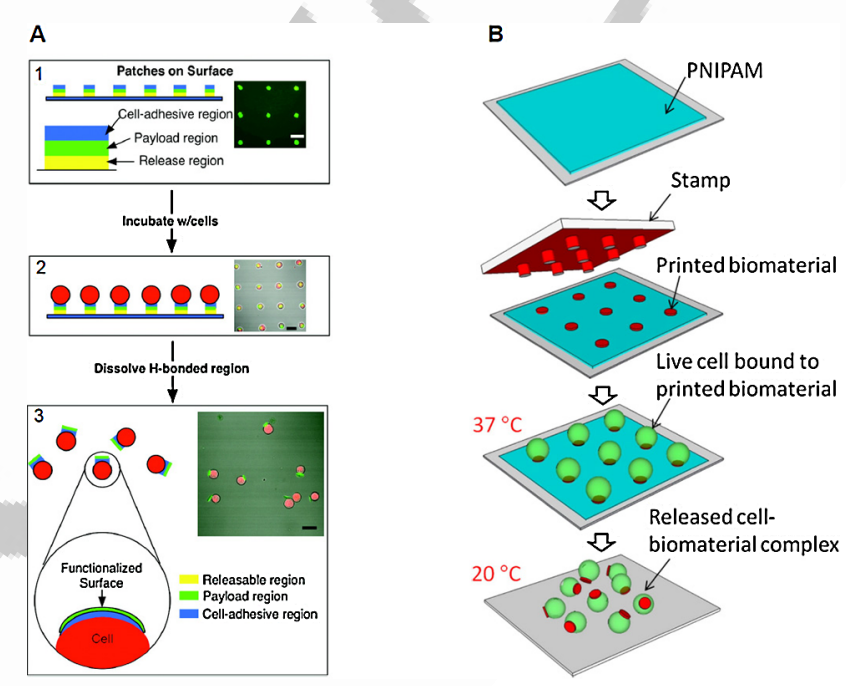

Figure 5. A) Overview of a cell functionalization scheme, with confocal images demonstrating each step. (1) A regular array of surface-bound patches spaced $50 \mu \mathrm{m}$ apart. The green fluorescence is from the FITC-PAH used to fabricate the payload region. After $\mathrm{CH} 27$ B-cell incubation and attachment (2), a majority $(85 \pm 3 \%)$ of the surface-bound patches are occupied. The red fluorescence is from CellTracker Red CMPTX, which nonselectively tags the interior of living cells. (3) After the temperature is reduced to $4{ }^{\circ} \mathrm{C}$ for $30 \mathrm{~min}$, the patches are released from the surface while remaining attached to the cell membrane. All scale bars are $25 \mu \mathrm{m}$. (Reprinted with permission from [41]. Copyright ๑ 2008 American Chemical Society.) B) Procedure of functionalizing or assembling live cells with microcontact-printed biomaterials using spin-coated PNIPAM as the sacrificial layer. Microparticles and single cells are used here as a model system. (Reprinted with permission from [44]. Copyright @ 2014 Elsevier.)

Overall the herein-described methods for cell functionalization confirm that cell-nanomaterials conjugations may act as an ideal system for cell function remodeling and drug release to effectively improve cell-based therapies.

\section{Goals of Cell Engineering}

\section{In vivo cell migration}

Cell based therapies have attracted increased attention in biomedicine. One of the greatest challenges in cell-based therapies is to minimally invasively deliver a large quantity of viable cells to the target tissue. For example the inefficient homing of systemically delivered MSCs, is caused predominantly by inade- 
quate expression of cell surface adhesion receptors. ${ }^{[45]}$ It was already reported in this review that covalently conjugated SLeX on MSC surface through a biotin-streptavidin bridge instructs cell rolling without altering the cell phenotype and the differentiation potential. The conjugation of SLeX on the MSC surface is stable, versatile, and induces a robust rolling response on P-selectin coated substrates. ${ }^{[24 b, 46]}$ It was also reported that the conjugation of stem cells with bispecific antibodies can be directly injected and retained by injured myocardium or targeted to injured myocardial tissues for tissue regeneration. ${ }^{[47,48]}$ These methods offer a simple approach to explore engineered cell homing and potentially target any cell type to specific tissues via the circulation.

\section{Cell-matrix and cell-cell interactions}

The precise arrangement of cells in their substrate is critical in controlling cell function. The modification of substrates has been widely explored for an accurate control of cell adhesion. More recently, many research groups have been focused their work in the modification of the cell surface in order to control cell fate and biointerfaces interactions. For instance, the functionalization of cells with short oligonucleotides promotes specific adhesive properties. Oligonucleotides offer several advantages, including highly specificity and ease of synthesis. Cellular lipid bilayers can be modified with oligonucleotides incorporating hydrophobic molecules at their $3^{\prime}$ or $5^{\prime}$ ends. ${ }^{[49-52]}$ Iwata and co-workers, prepared amphiphilic PEG-lipid polymers that were attached to specific oligonucleotide sequences. ${ }^{[53]}$ By incorporating complementary DNA sequences attached to amphiphilic PEG-lipids into the membranes of two cell populations, cell-cell or cell-substrate interactions were subsequently mediated via hybridization between the two complementary DNA sequences $A$ similar approach was recently described by Gartner and co-workers for programming the adhesive properties of cells independent of proteins, glycans, or their endogenous adhesion machinery. ${ }^{[54]}$ They develop a strategy to chemically control cell adhesion using membrane anchored singlestranded DNA oligonucleotides (Figure 6). Bertozzi and coworkers reported on non-covalent cell surface engineering as a strategy to display synthetic glycolpolymers that mimic cell surface mucins, a class of glycoproteins with roles in modulating cell-cell interactions. ${ }^{[11]}$ The same group demonstrated that the kinetics of the assembly process is controlled by adjusting the DNA sequence complexity, density, and cell density. Thus, cell assembly can be tuned, enabling the design of 3-dimensional microtissues with defined cell composition and stoichiometry. ${ }^{[55]}$ In an alternate approach Francis and co-workers demonstrated that cell adhesion events can be programmed through the attachment of synthetic ssDNA strands to the surfaces of living cells. ${ }^{[33,56]}$ DNA strands are used to anchor cells to specified locations on surfaces in a sequence-dependent fashion. Yousaf and co-workers developed a strategy to induce specific and stable cell-cell contacts through chemoselective cell-surface engineering based on liposome fusion that successfully displayed bioorthogonal functional groups on cell membrane. ${ }^{[57,58]}$ Such strategy allows for modulation of cell ad-

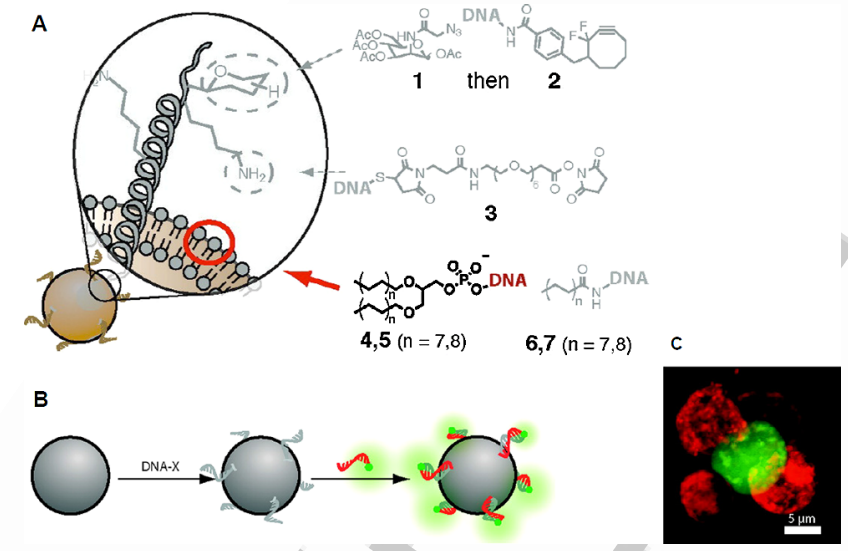

Figure 6. A) Cell-surface glycans are targeted for chemical remodeling following a 3 day incubation in azido sugar 1 and subsequent covalent modification with difluorocyclooctyne (DIFO, 2)-conjugated DNA. Protein lysine side chains are conjugated to $N$-hydroxysuccinimide ester-modified DNA 3. Fatty acid amides 6, 7 and dialkylphosphoglyceride-modified oligonucleotides 4, 5 (bold) target the lipid bilayer non-covalently. B) Incorporation of oliognucleotides to cell surfaces. Scheme for labeling and selectively quantifying cell-surface oligonucleotides by flow cytometry. C) Fluorescence activated cell sorting (FACS)-purified cell cluster imaged by confocal fluorescence microscopy. (Reprinted with permission from [54]. Copyright ๑ 2011 American Chemical Society.)

hesion, the generation of stable 3D spheroid and multilayered tissue-like structures. Since the ligation tether contains photoactive lipids, remote control of disassembly could be achieved upon UV light illumination. The use of sophisticated bioplotting systems could be employed in the future to assemble such aggregates of cells into complex 3D structures with a high geometrical precision.

\section{Control of cellular activity}

Cell surface engineering has been explored to control cellular functions such as proliferation and differentiation and drug delivery. A major limitation of cell therapies is the rapid decline in viability and function of the transplanted cells. Stimulatory biomolecules can be coupled to cells before transplantation to enhance their viability, proliferation and therapeutic potential. ${ }^{[27]}$ Likewise, the conjugation of drug-loaded nanoparticles to the surfaces of therapeutic cells provides sustained cell stimulation, enhancing the efficacy of cell therapies while minimizing the systemic side effects. ${ }^{[21,59]}$ Using such strategy, drug molecules may be slowly released from cell-bound nanoparticles and primarily recaptured by particle-carrying cells.

The direct deposition approach for cell coating is quite simple and straightforward method to modulate or control the response cells to their environment. ${ }^{[35-39,60]}$ Positively charged polymers can spontaneously bind to the outer-surface membrane of microorganism through electrostatic interactions. Successful application of LbL technology for encapsulation of cells will have a major impact on a diverse set of clinical fields including tissue engineering, novel therapeutic treatments, and targeted delivery. For instance, LbL coating of pancreatic islets was tested to avoid immune rejection after transplanta- 
tion. ${ }^{[36,37]}$ Moreover therapeutic molecules can be included within the LbL protecting shell to control cell function. ${ }^{[60]}$ For example, NSCs encapsulated with a IGF-1 loaded LbL coating, significantly enhanced the proliferation of the encapsulated cells, demonstrating a drug-carrier function of the LbL singlecell nanocoating. ${ }^{[40]}$

\section{Conclusions}

Cells interact with the extracellular environment through the molecular receptors and ligands present on the membrane. Research results reviewed in this paper clearly demonstrate that cell-cell and cell-ECM interactions can be remodeled by means of cell surface engineering. Cell surface engineering represents a powerful tool to manipulate living cells by decorating the cell membrane with specific molecules of interest and specialized structures, for example nanoparticles and patches. This provides an alternative to control the adhesion properties of living cells without a dependence on the receptors that they possess. The technologies herein described should also have broad implications on cellular therapies that utilize systemic administration and require targeting of cells to specific tissues, tissue engineering or drug delivery. In addition, these results suggest therapeutic cells are promising vectors for actively targeted drug delivery. Many issues remain to be addressed in cell surface engineering. The highly dynamic nature of the cell surface and the need of mild reaction conditions are still major challenges for living cell surface engineering. Nevertheless, despite the challenges, cell membrane engineering has emerged as an effective method to manipulate cell function and cellular nanomodification is a promising strategy for improving current and future cell-based therapeutic practices.

\section{Acknowledgments}

C.A.C. acknowledges funding support from the Portuguese Foundation for Science and Technology (FCT) (fellowship SFRH/BPD/100594/2014). This work was also supported by European Research Council grant agreement ERC-2014-ADG669858 for project ATLAS. — Keywords ok?

Keywords: cell membranes • ligands - liposomes nanoparticles $\cdot$ surface engineering

[1] F. Edalat, I. Sheu, S. Manoucheri, A. Khademhosseini, Curr. Opin. Biotech nol. 2012, 23, 820-825.

[2] C. A. Custódio, R. L. Reis, J. F. Mano, Adv. Healthcare Mater. 2014, 3, $797-810$.

[3] C. Frantz, K. M. Stewart, V. M. Weaver, J. Cell Sci. 2010, 123, 4195-4200

[4] W. F. Zheng, W. Zhang, X. Y. Jiang, Adv. Healthcare Mater. 2013, 2, $95-$ 108.

[5] A. Rodrigo-Navarro, P. Rico, A. Saadeddin, A. J. Garcia, M. Salmeron-Sanchez, Sci. Rep. 2014, 4, 5849

[6] K. Simons, J. L. Sampaio, Cold Spring Harbor Perspect. Biol. 2011, 3, a004697.

[7] L. K. Mahal, C. R. Bertozzi, Chem. Biol. 1997, 4, 415-422.

[8] B. Kellam, P. A. De Bank, K. M. Shakesheff, Chem. Soc. Rev. 2003, 32, $327-337$.
[9] M. D. Mager, V. LaPointe, M. M. Stevens, Nat. Chem. 2011, 3, 582-589.

[10] X. D. Xu, H. Cheng, W. H. Chen, S. X. Cheng, R. X. Zhuo, X. Z. Zhang, Sci. Rep. 2013, 3, $\square$ article number?

[11] D. Rabuka, M. B. Forstner, J. T. Groves, C. R. Bertozzi, J. Am. Chem. Soc. 2008, 130, 5947-5953.

[12] K. Simons, D. Lingwood, U. Coskun, M. Grzybek, FEBS J. 2009, 276, 5353.

[13] I. M. Verma, M. D. Weitzman, Annu. Rev. Biochem. 2005, 74, 711-738.

[14] W. A. Zhao, G. S. L. Teo, N. Kumar, J. M. Karp, Mater. Today 2010, 13, 1421.

[15] M. S. Penn, A. A. Mangi, Circ. Res. 2008, 102, 1471-1482.

[16] M. Kato, M. Mrksich, J. Am. Chem. Soc. 2004, 126, 6504-6505.

[17] J. H. Collier, M. Mrksich, Proc. Natl. Acad. Sci. USA 2006, 103, 2021-2025.

[18] M. Howarth, K. Takao, Y. Hayashi, A. Y. Ting, Proc. Natl. Acad. Sci. USA 2005, 102, 7583-7588.

[19] I. Chen, M. Howarth, W. Y. Lin, A. Y. Ting, Nat. Methods 2005, 2, 99-104.

[20] P. Wu, W. Q. Shui, B. L. Carlson, N. Hu, D. Rabuka, J. Lee, C. R. Bertozzi, Proc. Natl. Acad. Sci. USA 2009, 106, 3000-3005.

[21] M. T. Stephan, D. J. Irvine, Nano Today 2011, 6, 309-325.

[22] D. Y. Lee, S. J. Park, J. H. Nam, Y. Byun, Tissue Eng. 2006, 12, 615-623.

[23] Y. Krishnamachari, M. E. Pearce, A. K. Salem, Adv. Mater. 2008, 20, $989-$ 993.

[24] a) D. Sarkar, P. K. Vemula, D. P. Spelke, J. M. Karp, 2009 35th Annual Northeast Bioengineering Conference 2009, 68-69; b) D. Sarkar, J. A. Spencer, J. A. Phillips, W. A. Zhao, S. Schafer, D. P. Spelke, L. J. Mortensen, J. P. Ruiz, P. K. Vemula, R. Sridharan, S. Kumar, R. Karnik, C. P. Lin, J. M. Karp, Blood 2011, 118, e184-e191.

[25] W. A. Zhao, S. Schafer, J. Choi, Y. J. Yamanaka, M. L. Lombardi, S. Bose, A. L. Carlson, J. A. Phillips, W. S. Teo, I. A. Droujinine, C. H. Cui, R. K. Jain, J. Lammerding, J. C. Love, C. P. Lin, D. Sarkar, R. Karnik, J. M. Karp, Nat. Nanotechnol. 2011, 6, 524-531.

[26] P. Nacharaju, F. N. Boctor, B. N. Manjula, S. A. Acharya, Transfusion 2005, 45, 374-383.

[27] M. T. Stephan, J. J. Moon, S. H. Um, A. Bershteyn, D. J. Irvine, Nat. Med. 2010, 16, $1035-\mathrm{U} 1135$.

[28] C. D. Rillahan, A. Antonopoulos, C. T. Lefort, R. Sonon, P. Azadi, K. Ley, A. Dell, S. M. Haslam, J. C. Paulson, Nat. Chem. Biol. 2012, 8, 661-668.

[29] R. Xie, S. Hong, L. Feng, J. Rong, X. Chen, J. Am. Chem. Soc. 2012, 134, 9914-9917.

[30] A. Pulsipher, M. E. Griffin, S. E. Stone, J. M. Brown, L. C. Hsieh-Wilson, J. Am. Chem. Soc. 2014, 136, 6794-6797.

[31] D. S. Wade M. Fox, Micro- and Nanoengineering of the Cell Surface, Vol. 1, William Andrew, 2014.

[32] L. K. Mahal, K. J. Yarema, C. R. Bertozzi, Science 1997, 276, 1125-1128,

[33] R. A. Chandra, E. S. Douglas, R. A. Mathies, C. R. Bertozzi, M. B. Francis, Angew. Chem. Int. Ed. 2006, 45, 896-901; Angew. Chem. 2006, 118, 910 915.

[34] E. Saxon, C. R. Bertozzi, Science 2000, 287, 2007-2010.

[35] F. C. Picart, J.-C. Voegel, G. Decher, Layer-by-Layer Films for Biomedical Applications, Wiley, 2014.

[36] M. Germain, P. Balaguer, J. C. Nicolas, F. Lopez, J. P. Esteve, G. B. Sukhorukov, M. Winterhalter, H. Richard-Foy, D. Fournier, Biosens. Bioelectron. 2006, 21, 1566-1573.

[37] J. T. Wilson, W. Cui, V. Kozlovskaya, E. Kharlampieva, D. Pan, Z. Qu, V. R. Krishnamurthy, J. Mets, V. Kumar, J. Wen, Y. Song, V. V. Tsukruk, E. L. Chaikof, J. Am. Chem. Soc. 2011, 133, 7054-7064.

[38] S. Mansouri, Y. Merhi, F. M. Winnik, M. Tabrizian, Biomacromolecules 2011, 12, 585-592.

[39] N. G. Veerabadran, P. L. Goli, S. S. Stewart-Clark, Y. M. Lvov, D. K. Mills, Macromol. Biosci. 2007, 7, 877-882.

[40] W. Li, T. Guan, X. Zhang, Z. Wang, M. Wang, W. Zhong, H. Feng, M. Xing, J. Kong, ACS Appl. Mater. Interfaces 2015, 7, 3018-3029.

[41] A. J. Swiston, C. Cheng, S. H. Um, D. J. Irvine, R. E. Cohen, M. F. Rubner, Nano Lett. 2008, 8, 4446-4453.

[42] E. Castro, J. F. Mano, J. Biomed. Nanotechnol. 2013, 9, 1129-1136.

[43] H. Cheng, C. J. Kastrup, R. Ramanathan, D. J. Siegwart, M. L. Ma, S. R. Bogatyrev, Q. B. Xu, K. A. Whitehead, R. Langer, D. G. Anderson, ACS Nano 2010, 4, 625-631.

[44] Z. Wang, J. Xia, Y. Yan, A. C. Tsai, Y. Li, T. Ma, J. Guan, Acta Biomater 2015, 11, 80-87. 
[45] R. Sackstein, J. S. Merzaban, D. W. Cain, N. M. Dagia, J. A. Spencer, C. P. Lin, R. Wohlgemuth, Nat. Med. 2008, 14, 181-187.

[46] D. Sarkar, P. K. Vemula, G. S. L. Teo, D. Spelke, R. Karnik, L. Y. Wee, J. M. Karp, Bioconjugate Chem. 2008, 19, 2105-2109.

[47] a) L. G. Lum, H. Fok, R. Sievers, M. Abedi, P. J. Quesenberry, R. J. Lee Blood Cells Mol. Dis. 2004, 32, 82-87; b) R. J. Lee, Q. Z. Fang, P. A. Davol, Y. P. Gu, R. E. Sievers, R. C. Grabert, J. M. Gall, E. Tsang, M. S. Yee, H. Fok, N. F. Huang, J. F. Padbury, J. W. Larrick, L. G. Lum, Stem Cells 2007, 25, $712-717$.

[48] J. S. Yu, Y. K. Wu, Y. P. Gu, Q. Z. Fang, R. Sievers, C. H. Ding, J. E. Olgin, R. J. Lee, J. Cell. Mol. Med. 2015, 19, 1483-1491.

[49] G. G. Borisenko, M. A. Zaitseva, A. N. Chuvilin, G. E. Pozmogova, Nucleic Acids Res. 2009, 37, 口article number? $\square$.

[50] K. Borjesson, J. Tumpane, T. Ljungdahl, L. M. Wilhelmsson, B. Norden, T. Brown, J. Martensson, B. Albinsson, J. Am. Chem. Soc. 2009, 131, 2831 2839.

[51] T. Itagaki, Y. Arima, R. Kuwabara, N. Kitamura, H. Iwata, Colloids Surf. $B$ 2015, 135, 765-773.

[52] T. Matsui, Y. Arima, N. Takemoto, H. Lwata, Acta Biomater. 2015, 13, $32-$ 41.
[53] Y. Teramura, H. Chen, T. Kawamoto, H. Iwata, Biomaterials 2010, 31 , 2229-2235.

[54] N. S. Selden, M. E. Todhunter, N. Y. Jee, J. S. Liu, K. E. Broaders, Z. J. Gartner, J. Am. Chem. Soc. 2012, 134, 765-768.

[55] Z. J. Gartner, C. R. Bertozzi, Proc. Natl. Acad. Sci. USA 2009, 106, $4606-$ 4610.

[56] S. C. Hsiao, B. J. Shum, H. Onoe, E. S. Douglas, Z. J. Gartner, R. A. Mathies, C. R. Bertozzi, M. B. Francis, Langmuir 2009, 25, 6985-6991.

[57] D. Dutta, A. Pulsipher, W. Luo, M. N. Yousaf, J. Am. Chem. Soc. 2011, 133, $8704-8713$.

[58] W. Luo, A. Pulsipher, D. Dutta, B. M. Lamb, M. N. Yousaf, Sci. Rep. 2014 4, 口article number? $\square$

[59] W. Chen, L. W. Fu, X. Y. Chen, J. Controlled Release 2015, 219, 560-575. [60] V. Gribova, R. Auzely-Velty, C. Picart, Chem. Mater. 2012, 24, 854-869.

Manuscript received: February 2, 2016

Accepted Article published: $\square$ II, 0000

Final Article published: $\mathbf{\square} \mathbf{0}, 0000$ 


\section{REVIEW}

More than skin deep: This Focus Review highlights the recent progress on engineering cell surfaces with biological, chemical or physical methods to modulate cell functions and control cell-cell and cell-microenvironment interactions. $\square$ Please provide an $18 \times 18 \mathrm{~cm}$ frontispiece image with the proofs, and academic titles for all authors

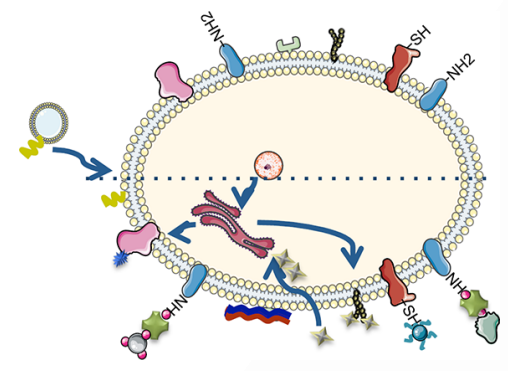

Biointerfaces

Catarina A. Custódio, João F. Mano* $\mathbf{\square}-\mathbf{\square}$

Cell Surface Engineering to Control Cellular Interactions

Focus Review from J. Mano @udominho on cell \#surface engineering SPACE RESERVED FOR IMAGE AND LINK

Share your work on social media! ChemNanoMat has added Twitter as a means to promote your article. Twitter is an online microblogging service that enables its users to send and read text-based messages of up to 140 characters, known as "tweets". Please check the pre-written tweet in the galley proofs for accuracy. Should you or your institute have a Twitter account, please let us know the appropriate username (i.e., @accountname), and we will do our best to include this information in the tweet. This tweet will be posted to the journal's Twitter account @ChemNanoMat (follow us!) upon online publication of your article, and we recommended you to repost ("retweet") it to alert other researchers about your publication.

Please check that the ORCID identifiers listed below are correct. We encourage all authors to provide an ORCID identifier for each coauthor. ORCID is a registry that provides researchers with a unique digital identifier. Some funding agencies recommend or even require the inclusion of ORCID IDs in all published articles, and authors should consult their funding agency guidelines for details. Registration is easy and free; for further information, see http:// orcid.org/.

Catarina A. Custódio http://orcid.org/0000-0003-2967-700X João F. Mano

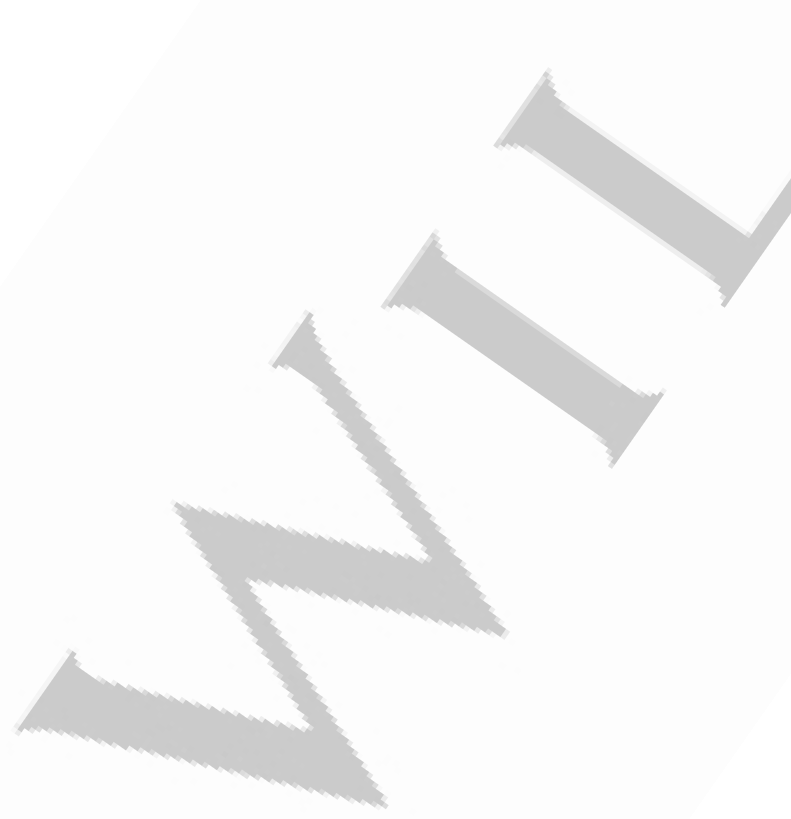

